\title{
Stronger Security Proofs for RSA and Rabin Bits
}

\author{
R. Fischlin and C.P. SchNorR \\ Fachbereich Mathematik/Informatik \\ Universität Frankfurt \\ PSF 111932 \\ 60054 Frankfurt/Main, Germany
}

\begin{abstract}
The RSA and Rabin encryption function are respectively defined as $E_{N}(x)=x^{e} \bmod N$ and $E_{N}(x)=x^{2} \bmod N$, where $N$ is a product of two large random primes $p, q$ and $e$ is relatively prime to $\varphi(N)$. We present a much simpler and stronger proof of the result of Alexi, Chor, GoldreIch and SchnorR [ACGS88] that the following problems are equivalent by probabilistic polynomial time reductions: (1) given $E_{N}(x)$ find $x ;(2)$ given $E_{N}(x)$ predict the least-significant bit of $x$ with success probability $\frac{1}{2}+\frac{1}{\text { poly }(n)}$, where $N$ has $n$ bits. The new proof consists of a more efficient algorithm for inverting the RSA/Rabinfunction with the help of an oracle that predicts the least-significant bit of $x$. It yields provable security guarantees for RSA-message bits and for the RSA-random number generator for moduli $N$ of practical size.
\end{abstract}

\section{Introduction}

Randomness is a fundamental computational resource and the efficient generation of provably secure pseudorandom bits is a basic problem. YAO [Y82] and BLUM, MICALI [BM84] have shown that perfect random number generators (RNG) exist under reasonable complexity assumptions. Some perfect RNG's are based on the RSA-function $E_{N}(x)=x^{e} \bmod N$ and the Rabin-function $E_{N}(x)=x^{2} \bmod N$, where the integer $N$ is a product of two large random primes $p, q$ and $e$ is relatively prime to $\varphi(N)=(p-1)(q-1)$. The corresponding RNG transforms a random seed $x_{0} \in[1, N)$ into a bit string $b_{1}, \ldots, b_{m}$ of arbitrary polynomial length $m=n^{O(1)}$ according to the recursion $x_{i}:=E_{N}\left(x_{i-1}\right), b_{i}:=$ $x_{i} \bmod 2$, where $N$ has $n$ bits. The security of these RNG's is related to a result of [ACGS88] that the RSA/Rabin-function can be inverted in polynomial time if one is given an oracle which predicts from given $E_{N}(x)$ the least-significant bit of $x$ with success probability $\frac{1}{2}+\frac{1}{\text { poly }(n)}$. While the ACGS-result shows that the RSA/Rabin RNG is perfect in an asymptotic sense the practicality of this result has been questionable as the transformation of attacks against these RNG's into a full inversion of the RSA/Rabin-function (resp. the factorization of $N$ ) is rather slow.

The main contribution of this paper is a much simpler and stronger proof of the ACGS-result. The new proof gives a more efficient algorithm for the inversion of the RSA/Rabin-function if one is given an oracle that predicts the 
least significant message bit. While the new method is primarily of theoretical interest, it yields a security guarantee for moduli $N$ of practical size. We extend our results to the Rabin-function $E_{N}(x)=x^{2} \bmod N$. The reduction from $E_{N^{-}}$ inversion, resp. factoring $N$, to prediction is particular efficient for the absolute Rabin-function $E_{N}^{a}(x)=\left|x^{2} \bmod N\right|$, where $|y|=\min (y, N-y)$.

Notation. Let $N$ be product of two large primes $p, q$. Let $\mathbb{Z}_{N}=\mathbb{Z} / N \mathbb{Z}$ be the ring of integers modulo $N$, and let $\mathbb{Z}_{N}^{*}$ denote the subgroup of invertible elements in $\mathbb{Z}_{N}$. We represent elements $x \in \mathbb{Z}_{N}$ by their least nonnegative residue in the interval $[0, N)$, i.e., $\mathbb{Z}_{N}=[0, N)$. We let $[a x]_{N} \in[0, N)$ denote the least nonnegative residue of $a x(\bmod N)$. We use $[a x]_{N}$ for arithmetic expressions over $\mathbb{Z}$ while the arithmetic over $a, x \in \mathbb{Z}_{N}=[0, N)$ is done modulo $N$. Let $n$ be the bit length of $N, 2^{n-1}<N<2^{n}$. For $x \in \mathbb{Z}$ we let $\ell(x)=x \bmod 2$ denote the leastsignificant bit of $x$. Let $e$ be relatively prime to $\varphi(N)=(p-1)(q-1), e \neq 1$. The RSA cryptosystem enciphers a message $x \in \mathbb{Z}_{N}$ into $E_{N}(x)=x^{e} \bmod N$. Let $O_{1}$ be an oracle running in expected time $T$ which, given $E_{N}(x)$ and $N$, predicts the least-significant bit $\ell(x)$ of $x$ with advantage $\varepsilon: \operatorname{Pr}_{x, w}\left[O_{1}\left(E_{N}(x)\right)=\ell(x)\right] \geq \frac{1}{2}+\varepsilon$, where the probability refers to random $x \in_{R}[0, N)$ and the internal coin tosses $w$ of the oracle. We assume that the time $T$ of the oracle also covers the $n^{2}$ steps for the evaluation of the function $E_{N}$. Throughout the paper we assume that $\varepsilon^{-1}$ and $n$ are powers of $2, n \geq 2^{9}$. We let $\lg$ denote the logarithm function with base 2. For a finite set $A$ let $b \in_{R} A$ denote a random element of $A$ that is uniformly distributed. All time bounds count arithmetic steps using integers with $\lg \left(n \varepsilon^{-1}\right)$ bits. We use integers of that size for counting the votes in majority decisions.

Our results. Consider the problem to compute from $E_{N}(x)$ and $N$ the message $x \in \mathbb{Z}_{N}$ with the help of the oracle $O_{1}$ but without knowing the factorization of $N$. The new method inverts $E_{N}$ by iteratively tightening approximations $u N$ of random multiples $[a x]_{N}$ with known multiplier $a$ via binary division. The basic idea is that $\left[\frac{1}{2} a x\right]_{N}=\frac{1}{2}[a x]_{N}$ for even $[a x]_{N},\left[\frac{1}{2} a x\right]_{N}=\frac{1}{2}\left([a x]_{N}+N\right)$ for odd $[a x]_{N}$. Thus we get from a rational approximation $u N$ to $[a x]_{N}$ and the least-significant bit $\ell(a x)$ a tighter approximation to $\left[\frac{1}{2} a x\right]_{N}$ :

$$
\left[\frac{1}{2} a x\right]_{N}-\frac{1}{2}(u+\ell(a x)) N=\frac{1}{2}\left([a x]_{N}-u N\right) \text {. }
$$

Without knowing $x$ we get $\frac{1}{2}(u+\ell(a x)) N$ from the multiplier $a$, the previous approximation $u N$ and $E_{N}(x)$. This in turn yields $E_{N}(a x)=E_{N}(a) E_{N}(x)$ and a guess $O_{1}\left(E_{N}(a x)\right)$ for $\ell(a x)$. Binary division without an oracle has already been used by Goldwasser, Micali, Tong [GMT82]. The method of binary division is more efficient than the gcd-method in [BCS83], [ACGS88]. In order to decipher $E_{N}(x)$ it guesses the least-significant bits and approximate locations of two random multiples $[a x]_{N},[b x]_{N}$ whereas the ged-method requires four random multiples. Most importantly, the number of oracle calls becomes nearly minimal.

In section 2 we present our basic algorithm that inverts the RSA-function $E_{N}$ in expected time $O\left(n^{2} \varepsilon^{-2} T+n^{2} \varepsilon^{-6}\right)$, where $T$ is the time and $\varepsilon$ the advantage of oracle $O_{1}$. The expectation refers to the internal coin tosses of $O_{1}$ and of the inversion algorithm. This greatly improves the [ACGS88]-time bound $O\left(n^{3} \varepsilon^{-8} T\right)$ for oracle RSA-inversion. The new time bound differentiates the costs induced by the oracle calls and the additional overhead. The oracle calls induce $O\left(n^{2} \varepsilon^{-2} T\right)$ 
steps, we call the $O\left(n^{2} \varepsilon^{-6}\right)$ other steps the additional overhead. We generalize our security result to the $j$-th least-significant message bit for arbitrary $j$. This generalization affects only the additional overhead of $E_{N}$-inversion, the number of oracle calls remains unchanged.

In section 3 we introduce the subsample majority rule, a trick that improves the efficiency of majority decisions. Suppose we are given pairwise independent 0,1 -valued votes that each has an advantage $\varepsilon$ in predicting the target bit $\ell\left(a_{t} x\right)$. A large sample size $m$ is necessary in order to make the error probability $\frac{1}{m e^{2}}$ of the majority decision sufficiently small. To reduce the computational costs of the large sample we only use a small random subsample of it. While the random subsample induces only a small additional error probability the time for the subsample majority decision reduces to the size of the small subsample. The large sample is only mentally used for the analysis, it does not enter into the computation. Using this trick we gain a factor $\frac{n}{\lg n}$ in the number of oracle calls and in the time for the inversion of $E_{N}$. The reduced number of oracle calls is optimal up to factor $O(\lg n)$.

In section 4 we process all possible locations for $[a x]_{N},[b x]_{N}$ much faster than trying them separately. This reduces the additional overhead in the time for RSA-inversion to $O\left(n^{2} \varepsilon^{-4} \lg \left(n \varepsilon^{-1}\right)\right)$.

In section 5 we give conclusions for the security of RSA-message bits and of the RSA-random number generator for moduli $N$ of practical size. These conclusions are preliminary as the additional overhead can be further reduced.

In section 6 we extend the oracle inversion algorithm to the Rabin-function $E_{N}$ and we derive a security guarantee for the $x^{2} \bmod N$ generator under the assumption that factoring is hard. The oracle inversion of the absolute Rabinfunction is as fast as that of the RSA-function. For the centered Rabin-function the inversion runs in time $O\left(n \varepsilon^{-4} \lg \left(n \varepsilon^{-1}\right) T\right)$. The latter improves the previous time bound $O\left(n^{3} \varepsilon^{-11} T\right)$ due to [VV84] in connection with [ACGS88].

\section{RSA-inversion by binary division}

We introduce a novel method for inverting the RSA-function without knowing the factorization of $N$ if one is given an oracle $O_{1}$ that predicts the leastsignificant message bit with non-negligible advantage $\varepsilon$. The algorithm RSAinversion is a simple version of the new method, that will be made more efficient by subsequent modifications. In order to invert $E_{N}(x)$ it picks two random multipliers $a, b$ and guesses the least-significant bits and the approximate locations for the message multiples $[a x]_{N},[b x]_{N}$. For $a_{t}:=a 2^{-t} \bmod N$ it iteratively constructs rational approximations $u_{t} N$ so that $\left|\left[a_{t} x\right]_{N}-u_{t} N\right| \leq \frac{\varepsilon N}{4 \cdot 2^{t}}$ for $t=1, \ldots, n$. To this end it uses the method of binary division explained in the introduction. From the approximation $u_{n} N$ to $\left[a_{n} x\right]_{N}$ we get the message $x=a_{n}^{-1}\left\lfloor u_{n} N+\frac{1}{2}\right\rfloor \bmod N$. The main work is to determine the bits $\ell\left(a_{t} x\right)$ by majority decision using the oracle $O_{1}$.

The majority decision for $\ell\left(a_{t} x\right)$ uses multipliers $a_{t}+i a_{t-1}+b$ that are pairwise independent. Recall that the arithmetic on $a, b, x, a_{t}$ is done modulo $N$. 
The algorithm determines an integer $w_{t, i}$ that most likely satisfies the equation $\left(a_{t}+i a_{t-1}+b\right) x=\left[a_{t} x\right]_{N}+i\left[a_{t-1} x\right]_{N}+[b x]_{N}-w_{t, i} N$, in which case we call $w_{t, i}$ correct. The $i$-th measurement guesses $\ell\left(a_{t} x\right)$ by evaluating $\ell$ for both sides of the latter equation. We guess $\ell$ for the left hand side via the oracle $O_{1}$ and we use that the right hand side is linear in $\ell\left(a_{t} x\right)$. The majority decision performs $m=\min \left\{2^{t}, 2 n\right\} \varepsilon^{-2}$ measurements, where $m$ and the set $A_{m}$ of integers $i$ is chosen as to optimize the trade-off between error probability and efficiency of the majority decision. The use of pairwise independent multipliers for majority decision is a crucial contribution of [ACGS88].

\section{RSA-inversion}

1. INPUT $E_{N}(x), N$

$t:=0 \quad\left(t\right.$ is the stage ), pick random integers $a, b \in_{R} \mathbb{Z}_{N}^{*} \subset[0, N)$, guess rational integers $u \in \frac{\varepsilon^{3}}{4}\left[0,4 \varepsilon^{-3}\right), v \in \frac{\varepsilon}{4}\left[0,4 \varepsilon^{-1}\right)$ satisfying

$$
\left|[a x]_{N}-u N\right| \leq \frac{\varepsilon^{3}}{8} N, \quad\left|[b x]_{N}-v N\right| \leq \frac{\varepsilon}{8} N
$$

Guess the least-significant bits $\ell(a x), \ell(b x), a_{0}:=a, u_{0}:=u$.

2. WHILE $t<n$ DO

$$
\begin{aligned}
& t:=t+1, a_{t}:=\frac{1}{2} a_{t-1} \bmod N, \quad u_{t}:=\frac{1}{2}\left(u_{t-1}+\ell\left(a_{t-1} x\right)\right), \\
& m:=\min \left\{2^{t}, 2 n\right\} \varepsilon^{-2} . \\
& A_{m}:=\{i|| 1+2 i \mid \leq m\}, \quad w_{t, i}:=\left\lfloor u_{t}+i u_{t-1}+v\right\rfloor \text { for all } i \in A_{m} .
\end{aligned}
$$

Majority decision

$$
\begin{gathered}
z:=\#\left\{\begin{array}{l|l}
i \in A_{m} & \begin{array}{l}
O_{1}\left(E_{N}\left(\left(a_{t}+i a_{t-1}+b\right) x\right)\right)= \\
i \ell\left(a_{t-1} x\right)+\ell(b x)-w_{t, i} N \bmod 2
\end{array}
\end{array}\right\} \\
\ell\left(a_{t} x\right):=\left[\begin{array}{ll}
\left.0 \text { if } z \geq \frac{m}{2} \text { and } 1 \text { otherwise }\right] \quad \text { END while }
\end{array}\right.
\end{gathered}
$$

3. OUTPUT $x:=a_{n}^{-1}\left\lfloor u_{n} N+\frac{1}{2}\right\rfloor \bmod N$

Correctness. If $\ell\left(a_{t} x\right)$ is always correctly determined the rational approximation $u_{t} N$ to $\left[a_{t} x\right]_{N}$ tightens from stage $t-1$ to stage $t$ by a factor $\frac{1}{2}$. As $a_{t}=\frac{1}{2} a_{t-1} \bmod N$ we have $\left[a_{t} x\right]_{N}=\frac{1}{2}\left[a_{t-1} x\right]_{N}$ for even $\left[a_{t-1} x\right]_{N}, \quad\left[a_{t} x\right]_{N}=$ $\frac{1}{2}\left(\left[a_{t-1} x\right]_{N}+N\right)$ for odd $\left[a_{t-1} x\right]_{N}$. Hence

$$
\left[a_{t} x\right]_{N}-u_{t} N=\left[a_{t} x\right]_{N}-\frac{1}{2}\left(u_{t-1}+\ell\left(a_{t-1} x\right)\right) N=\frac{1}{2}\left(\left[a_{t-1} x\right]_{N}-u_{t-1} N\right) .
$$

Probability of success. We call $w_{t, i}$ correct if $0 \leq\left[a_{t} x\right]_{N}+i\left[a_{t-1} x\right]_{N}+[b x]_{N}-$ $w_{t, i} N<N$. Correct $w_{t, i}$ satisfy the equation (as $N$ is odd we have $-w_{t, i} N=$ $\left.w_{t, i} \bmod 2\right): \quad \ell\left(\left(a_{t}+i a_{t-1}+b\right) x\right)=\ell\left(a_{t} x\right)+i \ell\left(a_{t-1} x\right)+\ell(b x)+w_{t, i} \bmod 2$.

In the majority decision we replace in this equation $\ell\left(\left(a_{t}+i a_{t-1}+b\right) x\right)$ by $O_{1}\left(E_{N}\left(\left(a_{t}+i a_{t-1}+b\right) x\right)\right)$, and we determine $\ell\left(a_{t} x\right)$ so that the equation holds for the majority of the $i \in A_{m}$. The algorithm succeeds if step 1 guesses correctly and if the majority decisions for $\ell\left(a_{t} x\right)$ are all correct. In this case we have $\left|\left[a_{n} x\right]_{N}-u_{n} N\right| \leq \frac{\varepsilon N}{4 \cdot 2^{n}}<\frac{1}{2}$ and thus $a_{n} x=\left\lfloor u_{n} N+\frac{1}{2}\right\rfloor \bmod N$ and the output is correct. All probabilities refer to the random pair $(a, b) \in_{R}\left(\mathbb{Z}_{N}^{*}\right)^{2}$ and to the coin tosses of the oracle. We use the conditional probability for the case that we are in the right alternative, where step 1 guesses correctly and the bits $\ell\left(a_{t} x\right)$ of previous stages have been correctly determined. 
Error probability of $w_{t, i}$. Let us denote $w_{t, i}^{\prime}=u_{t}+i u_{t-1}+v$ so that $w_{t, i}=$ $\left\lfloor w_{t, i}^{\prime}\right\rfloor$. In the right alternative we have by iteration of equation $(1)\left[a_{j} x\right]_{N}-$ $u_{j} N=2^{-j}\left([a x]_{N}-u N\right)$ for all $j \leq t$. Therefore and since $2^{-t} \varepsilon^{2}|1+2 i| \leq 1$ for $i \in A_{m}$ we have

$$
\left|\left[a_{t} x\right]_{N}+i\left[a_{t-1} x\right]_{N}+[b x]_{N}-w_{t, i}^{\prime} N\right| \leq \frac{\varepsilon}{8}\left(2^{-t} \varepsilon^{2}|1+2 i|+1\right) N \leq \frac{\varepsilon}{4} N .
$$

Hence $w_{t, i}$ is correct except that there exists an integer between $w_{t, i}^{\prime} N$ and $\left[a_{t} x\right]_{N}+i\left[a_{t-1} x\right]_{N}+[b x]_{N}$. Therefore $w_{t, i}$ errs with probability at most $\frac{\varepsilon}{4}$. By using the $m$ integers $i \in A_{m}$ instead of $i=1, \ldots, m$ we save a factor 2 in $|i|$ and in the error probability of $w_{t, i}$.

Error probability of the majority decision. The multipliers $\left(\frac{1}{2}+i\right) a+b$ are pairwise independent for $|i|<\frac{1}{2} \min (p, q)$ since the matrix of the $\mathbb{Z}_{N}$-linear transformation $\left[\begin{array}{l}1, \frac{1}{2}+i \\ 1, \frac{1}{2}+j\end{array}\right]\left[\begin{array}{l}a \\ b\end{array}\right]$ has determinant $j-i \neq 0 \bmod N$ and $(a, b)$ is random in $\left(\mathbb{Z}_{N}^{*}\right)^{2}$. A similar argument shows that the errors of the $w_{t, i}$ for $i \in A_{m}$ are pairwise independent if we are in the right alternative. The $i$-th measurement is correct iff

$$
O_{1}\left(E_{N}\left(\left(a_{t}+i a_{t-1}+b\right) x\right)\right)=\ell\left(a_{t} x\right)+i \ell\left(a_{t-1} x\right)+\ell(b x)+w_{t, i} \bmod 2 .
$$

This is the case if the oracle guesses correctly and $w_{t, i}$ is correct. The error of the $\mathrm{i}$-th measurement can be dominated by 0,1 -valued random variables $X_{i}$ with $\mathrm{E}\left[X_{i}\right]=\mathrm{E}\left[O_{1}\left(E_{N}\left(\left(a_{t}+i a_{t-1}+b\right) x\right)\right) \neq \ell\left(\left(a_{t}+i a_{t-1}+b\right) x\right)\right]+\mathrm{E}\left[w_{t, i}\right.$ errs $]$ so that the $X_{i}$ are pairwise independent for $i \in A_{m}$. Hence $\mathrm{E}\left[X_{i}\right] \leq \frac{1}{2}-$ $\frac{3}{4} \varepsilon, \operatorname{Var}\left[X_{i}\right] \leq \frac{1}{4}$.

A majority decision is correct iff the majority of the $m$ measurements is correct. A majority decision errs only if $\frac{1}{m} \sum_{i} X_{i}-\mu \geq \frac{3}{4} \varepsilon$, where $\mu:=\frac{1}{m} \sum_{i} \mathrm{E}\left[X_{i}\right]$. We apply Chebyshev's inequality to the $m$ pairwise independent error variables $X_{i}$ with $i \in A_{m}$.

\section{Chebyshev's inequality.}

$$
\operatorname{Pr}\left[\left|\frac{1}{m} \sum_{i} X_{i}-\mu\right| \geq \frac{3}{4} \varepsilon\right] \leq \sum_{i} \operatorname{Var}\left[X_{i}\right]\left(m \frac{3}{4} \varepsilon\right)^{-2} \leq \frac{4}{9 m \varepsilon^{2}} .
$$

By $m=\min \left\{2^{t}, 2 n\right\} \varepsilon^{-2}$ the majority decisions for $\ell\left(a_{t} x\right)$ errs with probability $\frac{4}{2^{1}}$ for $t \leq 1+\lg n$ and with probability $\frac{2}{9 n}$ for $t \geq 1+\lg n$. The majority decision for $t=1, \ldots, n$ have error probability $\sum_{t \geq 1} \frac{4}{2^{1} 9}+(2 n-\lg n) /(9 n) \leq \frac{4}{9}+\frac{2}{9}=\frac{2}{3}$.

Running time. We give an upper bound for the expected number of steps required to compute $x$ when given $E_{N}(x)$ and $N$. We separately count the steps of the oracle calls and the other steps which form the additional overhead.

The oracle is queried about $E_{N}\left(\left(a_{t}+i a_{t-1}+b\right) x\right)$ for $t=1, \ldots, n$ for the $i \in A_{m}$. The oracle calls depend on $a, b$ but not on $u, v, \ell(a x), \ell(b x)$. So we keep $a, b$ fixed while we try all possibilities for $u, \ldots, \ell(b x)$. As the algorithm has success rate $\frac{1}{3}$ and calls the oracle at most $m \leq 2 n \varepsilon^{-2}$ times per stage, there are in total at most $3 \cdot 2 n^{2} \varepsilon^{-2} T$ oracle calls.

Each majority decision contributes to the additional overhead at most $2 n \varepsilon^{-2}$ steps that are performed with all oracle replies given. The algorithm does not need the exact rational $u_{t}+v$ and merely computes $w_{t, i}=\left\lfloor u_{t}+v+i u_{t-1}\right\rfloor$ using 
$\lg \left(n \varepsilon^{-1}\right)+O(1)$ precision bits from $u_{t}+v$ and $i u_{t-1}$. We see that the additional overhead is at most the product of the following factors

1. \# of quadruples $(u, v, \ell(a x), \ell(b x))$

$$
\begin{gathered}
4^{2} \varepsilon^{-4} 2^{2} \\
n \\
2 n \epsilon^{-2} \\
3
\end{gathered}
$$

2. \# of stages

3. \# of steps per majority decision

4. the inverse of the success rate

Hence the additional overhead is at most $3 \cdot 2^{7} n^{2} \varepsilon^{-6}$, and thus the expected time for the inversion of $E_{N}$ is $3 n^{2} \varepsilon^{-2}\left(2 T+2^{7} \varepsilon^{-4}\right)$.

Using an oracle for the $j$-th least-significant message bit. The $j$-th leastsignificant message bit $\ell_{j}(x)$ is called secure if $E_{N}$ can be inverted in polynomial time via an oracle $O_{j}$ that predicts $\ell_{j}(x)$ when given $E_{N}(x)$. Let oracle $O_{j}$ predict $\ell_{j}(x)$ with advantage $\varepsilon$ in expected time $T$. With the oracle $O_{j}$ the RSA-inversion proceeds in a similar way as for $j=1$. It guesses initially $L_{j}(a x), L_{j}(b x) \in\left[0,2^{j}\right)$, the integers that consist of the $j$ least-significant bits of $[a x]_{N},[b x]_{N}$. A main point is that the majority decision for $\ell_{j}\left(a_{t} x\right)$ takes into account carry overs from the $j-1$ least-significant bits. The equation

$$
\begin{gathered}
L_{j-1}\left(\left(a_{t}+i a_{t-1}+b\right) x\right)+2^{j-1} \ell_{j}\left(\left(a_{t}+i a_{t-1}+b\right) x\right)=L_{j-1}\left(a_{t} x\right)+ \\
i L_{j-1}\left(a_{t-1} x\right)+L_{j-1}(b x)+2^{j-1}\left(\ell_{j}\left(a_{t} x\right)+i \ell_{j}\left(a_{t-1} x\right)+\ell_{j}(b x)\right)-w_{t, i} N \bmod 2^{j}
\end{gathered}
$$

holds for correct $w_{t, i}$. In order to predict $\ell_{j}\left(a_{t} x\right)$ we replace in this equation $\ell_{j}\left(\left(a_{t}+i a_{t-1}+b\right) x\right)$ by $O_{j}\left(E_{N}\left(\left(a_{t}+i a_{t-1}+b\right) x\right)\right)$ and we recover $L_{j-1}\left(\left(a_{t}+\right.\right.$ $\left.\left.i a_{t-1}+b\right) x\right), L_{j-1}\left(a_{t} x\right)$ and $L_{j-1}\left(a_{t-1} x\right)$ recursively from the initial values $L_{j}(a x), L_{j}(b x)$, the approximate lacations $u N, v N$ and $N$. We choose $\ell_{j}\left(a_{t} x\right)$ so that the equation holds for the majority of $i \in A_{m}$.

The time of the inversion algorithm does not change from the case $j=1$ to arbitrary $j$, except that the factor under 1 . increases to $2^{2 j} 4^{2} \varepsilon^{-4}$ as we have to guess $L_{j}(a x), L_{j}(b x) \in\left[0,2^{j}\right)$. Now the time bound for RSA-inversion via $O_{j}$ is $O\left(n^{2} \varepsilon^{-2}\left(T+2^{2 j} \varepsilon^{-4}\right)\right)$ while it is $O\left(2^{4 j} n^{3} \varepsilon^{-8} T\right)$ for the ACGS-algorithm. There is a double advantage in the new time bound. The factor $2^{4 j}$ decreases to $2^{2 j}$ and it only affects the additional overhead. The additional overhead can be reduced by the method in section 4 to $O\left(n^{2} \varepsilon^{-4} \lg \left(n \varepsilon^{-1}\right)\right)$.

\section{From pairwise to mutually independent votes.}

We introduce the subsample majority decision, a trick that reduces the number of oracle calls for RSA-inversion by a factor $\lg n / n$. Suppose we have $m$ pairwise independent 0,1 -valued random variables (votes) $V_{i}$ for $i \in A_{m}$ that have advantage $\varepsilon$ in predicting the target bit $\ell\left(a_{t} x\right)$. The error probability of a majority decision is $\frac{1}{m \varepsilon^{2}}$, so we need a large $m$ to make this error small. To reduce the computational costs of the large sample we only use a small random subsample consisting of $m^{\prime} \ll m$ votes that are selected uniformly at random. Now the votes of the subsample are mutually independent, even though the original votes are merely pairwise independent, and their advantage $\varepsilon^{\prime}$ is close to $\varepsilon$. While the subsample induces only a small additional error probability $\exp \left(-2 m^{\prime} \varepsilon^{\prime 2}\right)$ the time 
for the subsample majority decision is only $m^{\prime}$. The large sample only appears in the mental error analysis, it does not enter into the computation. We can even fix a random subset $A_{m^{\prime}}^{\prime} \subset A_{m}$ for all SMAJ-calls, where $A_{m}:=\{i|| 1+2 i \mid \leq m\}$ as in section 2. Theorem 3 in section 4 uses such a fixed subset $A_{m^{\prime}}$.

Subsample Majority Decision (SMAJ). Pick $\left(\nu(1), \ldots, \nu\left(m^{\prime}\right)\right) \in_{R}\left(A_{m}\right)^{m^{\prime}}$ and guess that $\ell\left(a_{t} x\right)$ is $\left[\sum_{i=1}^{m^{\prime}} V_{\nu(i)} \geq \frac{m^{\prime}}{2}\right]$.

As in section 2 let $X_{i}$ be the error of the vote $V_{i}$ so that $\mathrm{E}\left[X_{i}\right] \leq \frac{1}{2}-\frac{3}{4} \varepsilon$. We denote $\mu=\frac{1}{m} \sum_{i \in A_{m}} \mathrm{E}\left[X_{i}\right]$ and $\mu^{\prime}=\frac{1}{m} \sum_{i \in A_{m}} X_{i}$. Consider the case that $\left|\mu^{\prime}-\mu\right|<\frac{1}{4} \varepsilon$ which by Chebyshev's inequality holds except with probability $\frac{\left.\operatorname{Max}_{i} \operatorname{Var}^{2} X_{i}\right]}{m(\varepsilon / 4)^{2}} \leq \frac{4}{m \varepsilon^{2}}$. The SMAJ-rule errs in this case only if $\frac{1}{m^{\prime}} \sum_{i=1}^{m^{\prime}} X_{\nu(i)} \geq$ $\mu^{\prime}+\frac{1}{2} \varepsilon$. For fixed values $X_{i}$ with $i \in A_{m}$ the variables $X_{\nu(1)}, \ldots, X_{\nu\left(m^{\prime}\right)}$ are identically distributed and mutually independent with mean value $\mu^{\prime}$. So we use

Bernstein's law of large numbers. For random $\left(\nu(1), \ldots, \nu\left(m^{\prime}\right)\right) \in_{R}\left(A_{m}\right)^{m^{\prime}}$ : $\operatorname{Pr}\left[\frac{1}{m^{\prime}} \sum_{i=1}^{m^{\prime}} X_{\nu(i)} \geq \mu^{\prime}+\frac{1}{2} \varepsilon\right]<\exp \left(-2\left(\frac{1}{2} m^{\prime} \varepsilon\right)^{2}\right)$.

Proposition 1. If the errors $X_{i}$ of the votes are pairwise independent and $\mathrm{E}\left[X_{i}\right] \leq \frac{1}{2}-\frac{3}{4} \varepsilon$ then SMAJ errs with probability at most $\frac{4}{m \varepsilon^{2}}+\exp \left(-\frac{1}{2} m^{\prime} \varepsilon^{2}\right)$.

Proof. If $\frac{1}{m^{\prime}} \sum_{i=1}^{m^{\prime}} X_{\nu(i)} \geq \frac{1}{2}$ we either have $\left|\mu-\mu^{\prime}\right| \geq \frac{1}{4} \varepsilon$ or $\frac{1}{m^{\prime}} \sum_{i=1}^{m^{\prime}} X_{\nu(i)} \geq$ $\mu^{\prime}+\frac{1}{2} \varepsilon$. The first event has probability $\leq \frac{4}{m \varepsilon^{2}}$ and the second $\leq \exp \left(-2 m^{\prime}\left(\frac{1}{2} \varepsilon\right)^{2}\right)$.

RSA-inversion using the SMAJ-rule. Let us modify the stages $t \geq 4+\lg n$ of RSA-inversion so that at these stages the SMAJ-rule is used with $m=2^{4} \varepsilon^{-2} n$ and the multipliers $a_{t}+i a_{t-1}+b$ with $i \in A_{m}$ - at stages $t \leq 3+\lg n$ the set $A_{m}$ is too small for SMAJ. We apply Proposition 1 with this $m$ and $m^{\prime}=2 \varepsilon^{-2} \lg n$. Then $\frac{1}{2} m^{\prime} \varepsilon^{2}=\lg n>1.4426 \ln n$, and thus a single SMAJ-call at stage $t \geq 4+\lg n$ fails with probability $\frac{4}{m \varepsilon^{2}}+n^{-1.4426}<\frac{1}{3 n}$ for $n \geq 2^{9}$. All SMAJ-calls together fail with probability $\frac{4}{9}+\frac{1}{3}=\frac{7}{9}$. As the number of oracle calls and the additional overhead decrease by a factor $\lg n / n$ we get

Theorem 2. Using an oracle $O_{1}$ that, given $E_{N}(x)$ and $N$, predicts $\ell(x)$ with advantage $\varepsilon$ in time $T$, the RSA-function $E_{N}$ can be inverted in expected time $9 n(\lg n) \varepsilon^{-2}\left(T+2^{6} \varepsilon^{-4}\right)$.

A main point is that the number of oracle calls for RSA-inversion is at most $9 n \varepsilon^{-2} \lg n$, whereas the ACGS-algorithm requires $(64)^{3} \frac{\pi^{2}}{3} n^{3} \varepsilon^{-8}$ oracle calls, where $(64)^{3} \frac{\pi^{2}}{3} \approx 2^{19.7}$. We can further reduce the factor 9 in Theorem 2 by guessing upon initiation closer approximations $u N, v N$ - this merely increases the additional overhead. On the other hand the number of oracle calls is nearly minimal.

Oracle optimality. Goldreich [G96] observed that the number $9 n \varepsilon^{-2} \lg n$ of oracle calls in Theorem 2 is minimal up to a factor $O(\lg n)$. 


\section{Processing all possible locations together.}

We sketch a first step in reducing the additional overhead in the time for RSAinversion. So far RSA-inversion processes all pairs of locations $u N, v N$ separately. Together these pairs can be processed much faster. We simulate the algorithm RSA-inversion for fixed $a, b$ and for all $u \in \frac{\varepsilon^{3}}{4}\left[0,4 \varepsilon^{-3}\right), v \in \frac{\varepsilon}{4}\left[0,4 \varepsilon^{-1}\right)$ with all oracle replies $O_{i, t}:=O_{1}\left(E_{N}\left(\left(a_{t}+i a_{t-1}+b\right) x\right)\right)$ given. The majority decision sets $\ell\left(a_{t} x\right)$ to 0 iff the equation (2) holds for the majority of the $i \in A_{m^{\prime}}^{\prime}$.

$$
O_{i, t}=i \ell\left(a_{t-1} x\right)+\ell(b x)+\left\lfloor u_{t}+v+i u_{t-1}\right\rfloor \bmod 2 .
$$

The main work of RSA-inversion is to compute for all $\bar{u} \in \frac{\varepsilon^{3}}{2^{5} n}\left[0,2^{6} n \varepsilon^{-3}\right), u_{t-1}:=$ $2 u_{t} \bmod 1$, all $v$, all $t$ and $l=\left(l_{1}, l_{2}\right):=\left(\ell\left(a_{t-1} x\right), \ell(b x)\right) \in\{0,1\}^{2}$ :

$$
\Gamma(\bar{u}, v, l, t):=\#\left\{i \in A_{m^{\prime}}^{\prime} \mid \text { equation (2) holds with } \bar{u}=u_{t}, v, l, t\right\} \text {. }
$$

This requires some technical algorithms and a tedious analysis that are contained in the full version of this paper. A main point is to separate in equation (2) the influence of $u_{t}+v$ - we only use a few precision bits of $u_{t}+v-$ and that of $u_{t-1}$. A key observation is that counting the $i$ that satisfy equation (2) can easily be done simultaneously for $u_{t-1}$ and $u_{t-1}+\frac{1}{2}$ if we separately count even and odd $i$. By exploiting and extending these ideas we can prove

Theorem 3. If all pairs $(u, v)$ are processed together, the additional overhead in $R S A$-inversion requires at most expected time $O\left(n^{2} \varepsilon^{-4} \lg \left(n \varepsilon^{-1}\right)\right)$.

The additional overhead in Theorem 3 can be further reduced. We can discard all pairs $(u, v)$ for which $\Gamma(u, v, l, t) / m^{\prime}$ is not in the correct range of numbers that differ from $\frac{1}{2} \pm \varepsilon$ by at most $\frac{3}{4} \varepsilon$, where $\varepsilon$ is the exact advantage of $O_{1}$. Thus we can restrict the set of pairs $(u, v)$ to a small subset of $\frac{\varepsilon^{3}}{4}\left[0,4 \varepsilon^{-3}\right) \times \frac{\varepsilon}{4}\left[0,4 \varepsilon^{-1}\right)$.

\section{Security of RSA-message bits and of the RSA-RNG.}

An important question of practical interest is how to generate efficiently many pseudorandom bits that are provably good under weak complexity assumptions. Provable security for the RSA-RNG follows from Theorems 2 and 3 . Under the assumption that there is no breakthrough in algorithms for inverting the whole RSA-function Theorems 2 and 3 yield provable security for RSA-message bits and for the RSA-RNG for moduli $N$ of practical size $-n=1000$ and $n=5000$.

Practical security of RSA-message bits. For given $E_{N}(x)$ it is impossible to predict $\ell(x)$ with advantage $\frac{1}{100}$ within one MIP-year $\left(3.16 \cdot 10^{13}\right.$ instructions) or else the RSA-function $E_{N}$ can be inverted faster than is possible by factoring $N$ using the fastest known algorithm. For this we choose $T:=3.16 \cdot 10^{13}, n:=$ $1000, \varepsilon:=\frac{1}{100}$. As the $O$-constant in Theorem 3 is about $2^{10}$, Theorems 2 and 3 yield a time bound $3 \cdot 10^{22}$ for factoring $N$ that is clearly smaller than 
$10^{25.5} \approx L_{N}\left[\frac{1}{3}, 1.9\right]$, the time for the fastest known factoring algorithm, see the next paragraph.

Each of the 10 least-significant RSA-message bits is individually secure for RSA-moduli $N$ with 1000 bits. This is because we can - see the end of section 2 - invert $E_{N}$ in time $9 n \lg n \varepsilon^{-2} T+O\left(n^{2} \varepsilon^{-4} \lg \left(n \varepsilon^{-1}\right)\right)$ using an oracle $O_{j}$ that predicts the $j$-th message bit $\ell_{j}(x)$.

On the other hand the ACGS-result does not give any security-guarantee for moduli $N$ of bit length 1000 , not even against one-step attackers with $T=1$, as $2^{19.7} 1000^{3} 100^{8} \approx 8.5 \cdot 10^{30} \gg 10^{25.5}$.

The fastest known factoring method. The fastest known algorithm for factoring $N$ or for breaking the RSA cryptoscheme requires at least $L_{N}\left[\frac{1}{3}, 1.9\right]^{1+o(1)}$ steps, where $L_{N}[v, c]=\exp \left(c \cdot(\ln N)^{v}(\ln \ln N)^{1-v}\right) . L_{N}\left[\frac{1}{3}, 1.9\right]$ is the conjectured run time of the number field sieve method with Coppersmith's modification using several number fields [BLP93]. Factoring even a non-negligible fraction of random RSA-moduli $N$ requires $L_{N}\left[\frac{1}{3}, 1.9\right]$ steps by this algorithm.

Practical and provably secure random bit generation. Let $N=p \cdot q$ be a random RSA-modulus with primes $p, q, e$ an RSA-exponent and let $x_{0} \in_{R}$ $[0, N)$. The RSA-RNG produces from random seeds $\left(x_{0}, N\right)$ the bit string $b=$ $\left(b_{1}, \ldots, b_{m}\right)$ as

$$
x_{i}=x_{i-1}^{e} \bmod N, \quad b_{i}=x_{i} \bmod 2 \text { for } i=1, \ldots, m .
$$

A statistical test $A$ rejects $b$ at tolerance level $\varepsilon$ if for random $a \in_{R}\{0,1\}^{m}$

$$
\left|\operatorname{Pr}_{b}[A(b)=1]-\operatorname{Pr}_{a}[A(a)=1]\right| \geq \varepsilon \text {. }
$$

A tolerance level $\frac{1}{100}$ is considered to be sufficient for practical purposes.

Theorem 4. Let the RSA-RNG produce from random seeds $\left(x_{0}, N\right)$ of length $2 n$ an output $b=\left(b_{1}, \ldots, b_{m}\right)$ of length $m$. Every statistical test $A$, that rejects the output at tolerance level $\varepsilon$, yields an algorithm that inverts the whole $R S A$ function $E_{N}$ in expected time $9 n \lg n(m / \varepsilon)^{2} T(A)+O\left(n^{2}(m / \varepsilon)^{4} \lg (n m / \varepsilon)\right)$ for a non-negligible fraction of $N$.

Proof. Suppose the bit string $b \in\{0,1\}^{m}$ is rejected by some test $A$ in time $T(A)$ and tolerance level $\varepsilon$. By Yao's argument, see eg. [K97, section 3.5, Lemma $\mathrm{P} 1]$, and since the distribution of $b$ is shift-invariant, there is an oracle $O_{1}$, which given $E_{N}(x)$ and $N$, predicts $\ell(x)$ in time $T(A)+m n^{2}$ with advantage $\varepsilon / m$ for a non-negligible fraction of $N$. By Theorems 2 and 3 , and assuming that $T(A)$ dominates $m n^{2}$, we can invert $E_{N}$ in the claimed expected time.

Corollary 5. The RSA-random generator produces for $n=5000$ from random seeds $\left(x_{0}, N\right)$ of bit length $10^{4}$ at least $m=10^{7}$ pseudorandom bits that withstand all statistical tests doable with the 1995 world computing power at tolerance level $\frac{1}{100}$, or else the whole RSA-function $E_{N}$ can be inverted in less than $L_{N}\left[\frac{1}{3}, 1.9\right]$ steps for a non-negligible fraction of $N$. 
Proof. OdLyzko rates the 1995 yearly world computing power to $3 \cdot 10^{8}$ MIPyears, where a MIP-year corresponds to $3.16 \cdot 10^{13}$ instructions. Then $3 \cdot 10^{8}$ MIP-years correspond to $10^{22}$ instructions. By Theorem 4 with a $O$-constant of $2^{10}$ we can invert $E_{N}$ using less than $10^{48}$ steps while $L_{N}\left[\frac{1}{3}, 1.9\right]>3.7 \cdot 10^{50}$.

\section{The $x^{2} \bmod N$ generator and the Rabin-function.}

The $x^{2} \bmod N$ generator has been proved to be secure under the assumption that factoring integers is hard. Here we show that this even holds for moduli $N$ of practical size. The $x^{2}$ mod $N$ generator transforms a random seed $\left(x_{0}, N\right)$ into a bit string $\left(b_{1}, \ldots, b_{m}\right)$ as $x_{i}:=E_{N}\left(x_{i-1}\right), b_{i}:=\ell\left(x_{i}\right)$ for $i=1, \ldots, m$. Here $E_{N}$ is the Rabin-function, $N$ is a random Blum integer - a product of two primes $p, q$ that are congruent $3 \bmod 4-$ and $x_{0}$ is a random number in $\mathbb{Z}_{N}$. We distinguish three variants of this generator, the absolute, the centered and the uncentered RNG, according to the following variants of the Rabin-function:

- the absolute Rabin-function $E_{N}^{a}(x)=\left|x^{2} \bmod N\right| \in(0, N / 2)$,

- the centered Rabin-function $E_{N}^{\mathrm{c}}(x)=x^{2} \bmod N \in(-N / 2, N / 2)$,

- the uncentered Rabin-function $E_{N}^{u}(x)=x^{2} \bmod N \in[0, N)$.

The centered function $E_{N}^{c}$ outputs $x^{2} \bmod N$, the absolute smallest residue of $x^{2}$ modulo $N$ in $(-N / 2, N / 2)$ whereas $E_{N}^{u}$ outputs the residue in $[0, N)$. Historically the uncentered RNG has been introduced as the $x^{2} \bmod N$ generator [BBS86]. However, the absolute and the centered RNG coincide and are more natural than the uncentered RNG. We note that

$$
E_{N}^{c}(x)= \pm E_{N}^{a}(x), \quad E_{N}^{a}(x)=\left|E_{N}^{u}(x)\right|, \quad E_{N}^{u}(x) \in\left\{E_{N}^{c}(x), E_{N}^{c}(x)+N\right\},
$$
where $|y|=\min (y, N-y)$ for $y \in \mathbb{Z}_{N}=[0, N)$. Thus $E_{N}^{c}$ extends the output of $E_{N}^{a}$ by one bit, the sign.

The absolute and the centered $R N G$ coincide in the output. Let $x_{i}^{a}, x_{i}^{c}, x_{i}^{u}$ denote the integer $x_{i}$ in the $i$-th iteration with $E_{N}^{a}, E_{N}^{c}, E_{N}^{u}$ and input $x_{0}=x_{0}^{a}=$ $x_{0}^{c}=x_{0}^{u}$. Using $E_{N}^{c}(x)= \pm E_{N}^{a}(x)$ we see by induction on $i$ that $x_{i}^{c}= \pm x_{i}^{a}$ and $\ell\left(x_{i}^{c}\right)=x_{i}^{c} \bmod 2=x_{i}^{a} \bmod 2=\ell\left(x_{i}^{a}\right)$.

On the other hand the uncentered RNG is quite different. It outputs the xor of $\ell\left(x_{i}^{c}\right)$ and the sign-bit $\left[x_{i}^{c}>0\right]$. The uncentered RNG is less natural. Consider the group $\mathbb{Z}_{N}^{*}(+1)$ of elements in $\mathbb{Z}_{N}^{*}$ with Jacobi symbol $1 . \mathbb{Z}_{N}^{*}(+1)$ is a subgroup of $\mathbb{Z}_{N}^{*}$ of index 2 that contains the group $Q R_{N}$ of quadratic residues modulo $N$. We see from $-1 \in \mathbb{Z}_{N}^{*}(+1) \backslash Q R_{N}$ that $E_{N}^{a}$ permutes the set $S_{N}=\mathbb{Z}_{N}^{*}(+1) \cap[1, N / 2), E_{N}^{c}$ permutes the set $Q R_{N} \cap(-N / 2, N / 2)$ and $E_{N}^{u}$ permutes $Q R_{N} \cap(0, N)$. The whole point is that $\mathbb{Z}_{N}^{*}(+1)$ can be decided in polynomial time whereas $Q R_{N}$ may be difficult to decide. So $E_{N}^{a}$ permutes a nice set $S_{N}$ whereas $E_{N}^{c}, E_{N}^{u}$ permute complicated sets. It comes as no surprise that we get better security results for the absolute/centered RNG than for the uncentered one. 
Oracle inversion of the absolute Rabin-function. The algorithm RSAinversion can be directly extended from the RSA-function to the permutation $E_{N}^{a}$ acting on $S_{N}=\mathbb{Z}_{N}^{*}(+1) \cap[1, N / 2)$. This extension uses an oracle $O_{1}$ which given $E_{N}^{a}(x)$ and $N$ predicts for random $x \in \mathbb{Z}_{N}^{*}(+1)$ the bit $\ell(x)$ with advantage $\varepsilon$. A main point is that the majority decisions must use multipliers $\bar{a}=a_{t}+i a_{t-1}+b$ in $\mathbb{Z}_{N}^{*}(+1)$ as we can only interpret the oracle for such inputs $E_{N}(\bar{a} x)$ with $x \in \mathbb{Z}_{N}^{*}(+1)$. On the average half of the multipliers $\bar{a}$ are in $\mathbb{Z}_{N}^{*}(+1)$, the usable multipliers are nearly uniformly distributed, see[P92]. For compensation the inversion algorithm guesses initially an approximate location $u N$ for $[a x]_{N}$ of half the previous distance. This doubles the additional overhead, but does not affect the number of oracle calls. With these remarks Theorems 2 and 3 extend from the RSA-function to the absolute Rabin-function $E_{N}^{a}$, Theorem 4 and Corollary 5 extend from the RSA-RNG to the absolute/centered $x^{2} \bmod N$ generator. The extended results prove security if factoring integers is hard, as the problems of inverting $E_{N}^{a}$ and of factoring $N$ are equivalent.

Theorem 6. The assertions of Theorems 2 and 3 hold for the absolute Rabinfunction $E_{N}^{a}$ in place of the RSA-function $E_{N}$. Theorems 4 and Corollary 5 hold for the absolute/centered $x^{2} \bmod N$ generator in place of the RSA-generator.

Comparison with the muddle square method. It is interesting to compare the centered $x^{2} \bmod N$ generator with the randomized $x^{2} \bmod N$ generator proposed by GoLDREICH and LEVIN [GL89,L93]: iteratively square $x_{i} \bmod N$ and output the scalar products $b_{i}=\left\langle x_{i}, z\right\rangle \bmod 2$ for $i=1, \ldots, m$ with a random bit string $z$. Following [GL89, L93] KNUTH shows that $N$ can be factored in expected time $O\left(n^{2} \varepsilon^{-2} m^{2} T(A)+n^{4} \varepsilon^{-2} m^{3}\right)$ for a non-negligible fraction of the $N$ if we are given a statistical test $A$ that rejects $\left(b_{1}, \ldots, b_{m}\right)$ at tolerance level $\varepsilon$, see [K97, section 3.5, Theorem P]. This yields a security guarantee for the muddle square method that is similar to the one of Corollary 5.

The problem of inverting of the (un)centered Rabin-function. Consider the permutations $E_{N}^{c}, E_{N}^{u}$ acting on the set of quadratic residues. The problems of inverting $E_{N}^{c}$ and $E_{N}^{u}$ are equivalent as we can easily transform one output into the other using that $E_{N}^{u}(x)-E_{N}^{c}(x) \in\{0, N\}$. We consider the oracle inversion of $E_{N}^{c}$. The problem we face in the oracle inversion of $E_{N}^{c}$ is that for given $\pm y \in \mathbb{Z}_{N}^{*}(+1)$ we do not know which of $\pm y$ is in $Q R_{N}$. A solution has been found by VAZIRANI and VAZIRANI [VV84]. We can determine the quadratic character of $\pm y$ using the oracle that predicts $\ell(z)$ for the inverse image $z \in Q R_{N}$ with $E_{N}^{c}(z)= \pm y$.

Let $O_{1}$ be an oracle which, given $E_{N}^{c}(x)$ and $N$, predicts the least-significant bit of $x \in Q R_{N}$ with advantage $\varepsilon, \operatorname{Pr}_{x, w}\left[O_{1}\left(E_{N}^{c}(x)\right)=\ell(x)\right] \geq \frac{1}{2}+\varepsilon$ for $x \in_{R}$ $Q R_{N}$ and the coin tosses $w$ of $O_{1}$. The main problem in extending the RSAinversion to the Rabin-function is that we can only use multipliers $\bar{a}=a_{t}+$ $i a_{t-1}+b$ that are in $Q R_{N}$ as we can only interpret oracle values $O_{1}\left(E_{N}^{c}(\bar{a} x)\right)$ with $\bar{a} x \in Q R_{N} . Q R_{N}$ is a subgroup of $Z_{N}^{*}$ with index 4 . 
Let $u$ s first suppose that 2 is in $Q R_{N}$ and that we are given $n \varepsilon^{-2}$ multipliers in $Q R_{N}$ of each of the two types $\left(\frac{1}{2}+i\right) a+b$ and $\frac{1}{2}(i a+b)$. Hereafter we show how to get rid of this assumption.

Inverting the centered Rabin-function. We describe how the algorithm differs from RSA-inversion if $2 \in Q R_{N}$.

Initially pick random $a, b \in_{R} \mathbb{Z}_{N}^{*}$ and produce about $n \varepsilon^{-2}$ quadratic residues of either type $\left(\frac{1}{2}+i\right) a+b, \frac{1}{2}(i a+b)$ - with $|1+2 i| \leq 4 m \varepsilon^{-2}-$ in $Q R_{N}$. On the average there are $n \varepsilon^{-2}$ residues in $Q R_{N}$ of either type. Guess the closest approximations $u N, v N$ to $[a x]_{N},[b x]_{N}$ with $u \in \frac{\varepsilon^{3}}{2^{4}}\left[0,2^{4} \varepsilon^{-3}\right), v \in \frac{\varepsilon}{4}\left[0,4 \varepsilon^{-1}\right)$.

At stage $t$ determine $\ell\left(\frac{1}{2} a x\right)$ by majority decision using oracle $O_{1}$ and all sample points $\left(\frac{1}{2}+i\right) a+b \in Q R_{N}$. Given $\ell\left(\frac{1}{2} a x\right)$ we can in the same way determine $\ell\left(\frac{1}{2} b x\right)$ using the sample points $\frac{1}{2}(i a+b)$ in $Q R_{N}$. Then replace $a, b$ by $\frac{1}{2} a \bmod N$, $\frac{1}{2} b \bmod N$ and go to the next stage. The new sample points $\left(i+\frac{1}{2}\right) a+b$ and $\frac{1}{2}(i a+b)$ are again in $Q R_{N}$ since we only divide by the quadratic residue 2 .

The case that 2 is a quadratic nonresidue. In this case we determine the quadratic residues $\left(\frac{1}{2}+i\right) a+b$ and $\frac{1}{2}(i a+b)$ at stages $t=1$ and $t=2$. We use the quadratic residues of stage 1 at the odd stages and the quadratic residues of stage 2 at the even stages. This is possible since we divide the residues by a power of 4 compared to stages 1 and 2 .

Determining quadratic residuosity. Suppose $\vec{a} \in \mathbb{Z}_{N}^{*}$ has Jacobi symbol 1 , then we have $\bar{a} \in Q R_{N}$ iff $\operatorname{Pr}_{z}\left[O_{1} E_{N}^{c}(\bar{a} z)=\ell(\bar{a} z)\right] \geq \frac{1}{2}+\varepsilon$ for $z \in \epsilon_{R} Q R_{N}$. This yields an oracle that predicts quadratic residuosity with advantage $\varepsilon$.

The algorithm for inverting the Rabin-function requires $O\left(n \varepsilon^{-4} \lg \left(n \varepsilon^{-1}\right) T\right)$ extra steps for the determination of the quadratic residues $\left(\frac{1}{2}+i\right) a+b, \frac{1}{2}(i a+$ $b)$. There is an extra factor 4 induced by the density $\frac{1}{4}$ of $Q R_{N}$ in $\mathbb{Z}_{N}^{*}$. To compensate for the smaller density the inversion algorithm guesses initially an approximate location $u N$ for $[a x]_{N}$ with $\frac{1}{4}$ times the previous distance. We reduce the additional overhead by the method of section 4 . Assuming that $T$ dominates $n$ we get

Theorem 7. The centered Rabin-function $E_{N}^{c}$ can be inverted in expected time $O\left(n \varepsilon^{-4} \lg \left(n \varepsilon^{-1}\right) T\right)$ with the help of an oracle that predicts $\ell(x)$ with advantage $\varepsilon$ in time $T$ when given $N$ and $E_{N}^{c}(x)$.

Conclusion. We have given a stronger security proof for RSA/Rabin bits. Our proof yields provable security for RSA-message bits, for the RSA-RNG and for the centered $x^{2} \bmod N$ generator for moduli $N$ of practical size, e.g. of bit length 1000 and 5000 . For the first time this yields provably secure and practical RNG's under the assumption that factoring integers is hard. On the other hand there are more efficient and provably secure RNG's based on stronger complexity assumptions, e.g. [MS91], [FS96].

Acknowledgement. We gratefully acknowledge the comments of D.E. Knuth and that of an anonymous referee that led to a considerably improved presentation of the material. 


\section{References}

[ACGS88] W. Alexi, B. Chor, O. Goldreich and C.P. Schnorr: RSA and Rabin Functions: certain parts are as hard as the whole. Siam J. Comp. 17 (1988), pp. 194-209.

[BCS83] M. Ben-Or, B. Chor and A. Shamir: On the Cryptographic Security of Single RSA-Bits. Proc. 15th ACM Symp. on Theory of Computation, April 1983, pp. 421-430.

[BBS86] L. Blum, M. Blum and M. Shub: A Simple Unpredictible Pseudo-Random Number Generator. Siam J. Comp. 15 (1986), pp. 364-383.

[BLP93] J.P. Buhler, H.W. Lenstra, Jr. and C. Pomerance: Factoring Integers with the Number Field Sieve. in: The Development of the number field sieve, (Ed. A.K. Lenstra, H.W. Lenstra, Jr.) Springer LNM 1554 (1993), pp. 50-94.

[BM84] M. Blum and S. Micali: How to Generate Cryptographically Strong Sequences of Pseudorandom Bits. Siam J. Comp., 13 (1984), pp. 850-864.

[FS96] J.B. Fischer and J. Stern: An Efficient Pseudo-Random Generator Provably as Secure as Syndrome Decoding. Proc. EUROCRYPT'96, Springer LNCS 1070 (1996) pp. 245-255.

[G96] O. Goldreich: personal information at the Oberwolfach workshop on Complexity Theory, November 10-16, 1996.

[GL89] O. Goldreich and L.A. Levin: Hard Core Bit for any One Way Function. Proc. of ACM Symp. on Theory of Computing (1989) pp. 25-32.

[GMT82] S. Goldwasser, S. Micali and P. Tong: Why and How to Establish a Private Code on a Public Network. Proc. 23rd IEEE Symp. on Foundations of Computer Science, Nov. 1982, pp. 134-144.

[HSS93] J. Hastad, A.W. Schrift and A. Shamir: The Discrete Logarithm Modulo a Composite Hides $O(n)$ bits. J. of Computing and Systems Science 47 (1993), pp. 376-404.

[K97] D.E. Knuth: Seminumerical Algorithms, 3rd edn. Addison-Wesley, Reading, MA (1997). Also Amendments to Volume 2. January 1997. http://www-cs-staff.Stanford.EDU/uno/taocp.html

[L93] L.A. Levin: Randomness and Nondeterminism. J. Symbolic Logic 58 (1993), pp. 1102-1103.

[MS91] S. Micali and C.P. Schnorr: Efficient, Perfect Polynomial Random Number Generators. J. Cryptology 3 (1991), pp. 157-172.

[095] A.M. Odlyzko: The Future of Integer Factorization. CryptoBytes, RSA Laboratories, 1 (1995), pp. 5-12.

[P92] R. Peralta: On the Distribution of Quadratic Residues and Non-residues Modulo a Prime Number. Math. Comp., 58

[R79] M.O. Rabin: Digital signatures and public key functions as intractable as factorization. TM-212, Laboratory of Computer Science, MIT, 1979.

[RSA78] R.L. Rivest. A. Shamir and L. Adleman: A Method for Obtaining Digital Signatures and Public Key Cryptosystems. Comm. ACM, 21 (1978), pp. 120-126.

[VV84] U.V. Vazirani and V.V. Vazirani: Efficient and Secure Pseudo-Random Number Generation. In Proc. 25th Symp. on Foundations of Computing Science (1984) IEEE, pp. 458-463.

[Y82] A.C. Yao: Theory and Application of Trapdoor Functions. Proc. of IEEE Symp. on Foundations of Computer Science (1982), pp. 80-91. 